\title{
Novel C stain-based chemical method for differentiating real and forged fingerprints
}

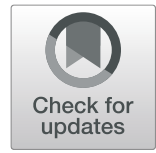

Sameer Saharan ${ }^{1}$, A. K. Yadav ${ }^{2}$ and Bhuvnesh Yadav ${ }^{1 *}$

\begin{abstract}
Background: Fingerprints are useful evidence for establishing identities. Development and detection of fingerprints are of immense help in criminal investigation. However, forged fingerprints identical to the real ones are emerging as a worldwide problem. Existing methods for development of fingerprints (powder method/iodine fuming method/ninhydrin test/ $\mathrm{AgNO}_{3}$ ) fail to distinguish between real and forged fingerprints when forged fingerprints are fortified with salts and amino acids. The present study was conducted with the objective to test applicability of $C$ stain for real and forged fingerprint differentiation.

Methodology: $C$ stain was applied on real and forged fingerprints in combination with conventional methods and was evaluated on the basis of development and differentiation of real and forged fingerprints.

Results: The proposed technique is successful in differentiating between real and forged fingerprints. Colour difference between real and forged fingerprints was observed by taking a combination of C stain with ninhydrin, black powder and iodine fuming, one at a time.

Conclusion: $\mathrm{C}$ stain method is an effective technique for distinguishing forged fingerprints from the real ones. It works as a distinction tool even when used in combination with existing development methods.
\end{abstract}

Keywords: C stain, Forensic Science, Forged fingerprints, Non-porous surface, Porous surface, Real fingerprints

\section{Introduction}

Fingerprint technology has been used in forensic science and practiced for more than hundred years now. Due to its uniqueness, fingerprint identification is one of the most widely used biometric techniques. However, it is important to elucidate the use of forgery of fingerprints for the fabrication of evidence involving fake use of genuine marks (Bonebreak 1976). Forged fingerprints are generally used by individuals who intend to commit a crime and then employ forged fingerprints to frame an innocent person or to divert attention of investigating agencies (Champod and Espinoza 2014).

According to a survey of 152 forensic professionals conducted by Geller et al. (2001), 85\% of them were aware of

\footnotetext{
* Correspondence: bhuvneshyadav@gmail.com

'Department of Chemistry, Biochemistry and Forensic Science, Amity School of Applied Sciences, Amity University Haryana, Gurugram, Haryana 122413, India

Full list of author information is available at the end of the article
}

the possibility to forge fingerprints. Further, $57 \%$ indicated that the threat was credible, and $45 \%$ admitted their inability to distinguish genuine fingerprints from forged fingerprints. In another study, Champod and Espinoza (2014) investigated the risks posed by forgeries and inability of forensic practitioners to detect them. In their study, practitioners reported more than 53\% forged fingerprints as genuine and $45 \%$ of genuine fingerprints as forged.

Powder method is the most commonly used approach for development of latent fingerprints with the help of glass fibre or camel hair brush (Sodhi and Kaur 2001). Other commonly used methods include ninhydrin, $\mathrm{AgNO}_{3}$ and iodine fuming (Oden and Von Hofsten 1954; Lennard et al. 1986; Bassam et al. 1991; Jasuja et al. 2012; Somanchi 2018). Several methods exist for preparation of forged fingerprints and are easily available online. This along with easy availability of raw material facilitates development of forged fingerprints to bypass biometric scanners or to implant on a crime scene with 
an aim to frame an innocent person. In the recent past, a sharp increase in fingerprint forgery has been observed in the form of Aadhaar frauds (Choudhari 2018; Saldanha 2018; Somanchi 2018), biometric manipulations (Qazi 2017), SIM card forgery and manual transfer of the fingerprint at documents and crime scene (LeGrand 2010). The conventional chemical methods (based on amino acids/salt binding) can differentiate real and PVA-based fingerprints but fail to differentiate when PVA-based fingerprints are fortified with amino acids and salts (Harvey et al. 2010).

Polyvinyl acetate (PVA) is commonly used material to develop forged fingerprints, due to its easy availability, low-cost and flexible end-product. The forged fingerprints are developed by taking a negative print on molten silicon. Recent trends show that forged fingerprints are being developed by incorporating artificial sweat in the fingerprint (Harvey et al. 2010). As most of the conventional fingerprint development methods are based on amino acid/salt-based technique (Lennard et al. 1986; Bassam et al. 1991; Jasuja and Singh 2009; Lee and Gaensslen 2010; Jasuja et al. 2012), fraudsters incorporate salts and amino acids in the forged fingerprints to mimic real fingerprints and avoid forgery detection during fingerprint analysis. Therefore, there is a timely need to develop a method which can distinguish forged fingerprints from the original ones. The present study was an attempt to differentiate real and fortified polyvinyl acetate (PVA)-based forged fingerprint with the help of a newly established protocol and to analyse its applicability and limitations.

\section{Materials and methods}

Negative imprints of the fingerprints of participants were taken on silicon mould, and forged fingerprints were developed with PVA (fortified with amino acids and salts) (Sousedik and Busch 2014). The fingerprints (real and PVA based) were analysed with $\mathrm{C}$ stain and other existing methods (powder method/ $\mathrm{AgNO}_{3} /$ ninhydrin/iodine fuming), in terms of variation in patterns, colour difference, impact of aging of fingerprints and type of surface (porous and non-porous). The plain latent fingerprints on three porous surfaces (paper, cardboard and wood) and three non-porous surfaces (chromogenic paper, plastic bottle and glass) were stored at room temperature with $50-60 \%$ humidity.

\section{Preparation of $\mathrm{C}$ stain \\ Step 1: preparation of stock solutions}

Solution 1: Aluminium chloride solution was prepared by adding $40 \mathrm{~g}$ of $\mathrm{AlCl}_{3}$ to $100 \mathrm{ml}$ water.

Solution 2: Calcium chloride solution was prepared by adding $100 \mathrm{~g}$ of $\mathrm{CaCl}_{2}$ to $150 \mathrm{ml}$ water.

Solution 3: Zinc chloride solution was prepared by adding $25 \mathrm{ml}$ of water to $50 \mathrm{~g}$ of dry $\mathrm{ZnCl}_{2}$.

Solution 4: Iodide-iodine solution was prepared by dissolving $0.90 \mathrm{~g}$ of dry Potassium iodide (KI) and $0.65 \mathrm{~g}$ of dry iodine in $50 \mathrm{ml}$ water.

\section{Step 2: preparation of $C$ stain}

A working solution was prepared by mixing $20 \mathrm{ml}$ of solution $1,10 \mathrm{ml}$ of solution $2,10 \mathrm{ml}$ of solution 3 and $12.5 \mathrm{ml}$ of solution 4 in a sterile beaker. The solution was transferred in a dark vessel and allowed the precipitate to settle for $12-24 \mathrm{~h}$. The clear solution was pipetted out in a dark bottle and stored with a leaf of iodine (Adamopoulos and Oliver 2006).

Present study was conducted on porous as well as non-porous surfaces, and its results were compared with results from conventional methods. Fingerprints were developed on three porous surfaces (paper, cardboard and wood) and three non-porous surfaces (chromogenic paper, plastic bottle and glass). Latent fingerprints (real and forged) were developed by the conventional methods (powder method, ninhydrin, iodine fuming, $\mathrm{AgNO}_{3}$ ) followed by $\mathrm{C}$ stain and vice versa (Table 1).

Amino acids and salts present in sweat start to decay with time, which makes it challenging to pick up

Table 1 Procedure for development and distinction of fingerprints by combining C stain method with conventional methods

\begin{tabular}{|c|c|c|}
\hline S. No. & Combinations & Procedure \\
\hline 1.1 & Powder method followed by C stain & First activated charcoal powder applied on latent prints and then $C$ stain was applied \\
\hline 1.2 & C stain followed by powder method & C stain method was applied on latent prints followed by activated charcoal powder \\
\hline 1.3 & Ninhydrin followed by C stain & Latent prints developed by ninhydrin followed by $C$ stain application \\
\hline 1.4 & C stain followed by ninhydrin & Application of C stain method on latent prints and then ninhydrin method was used \\
\hline 1.5 & $\mathrm{AgNO}_{3}$ followed by $\mathrm{C}$ stain & Development of latent prints with $\mathrm{AgNO}_{3}$ followed by application of $\mathrm{C}$ stain method \\
\hline 1.6 & C stain followed by $\mathrm{AgNO}_{3}$ & $\mathrm{C}$ stain method applied on latent prints and then $\mathrm{AgNO}_{3}$ was used for development \\
\hline 1.7 & lodine fuming followed by $C$ stain & Development of latent prints by iodine fumes followed by application of $\mathrm{C}$ stain \\
\hline 1.8 & C stain followed by lodine fuming & C stain method applied on latent prints followed by iodine fuming technique \\
\hline
\end{tabular}



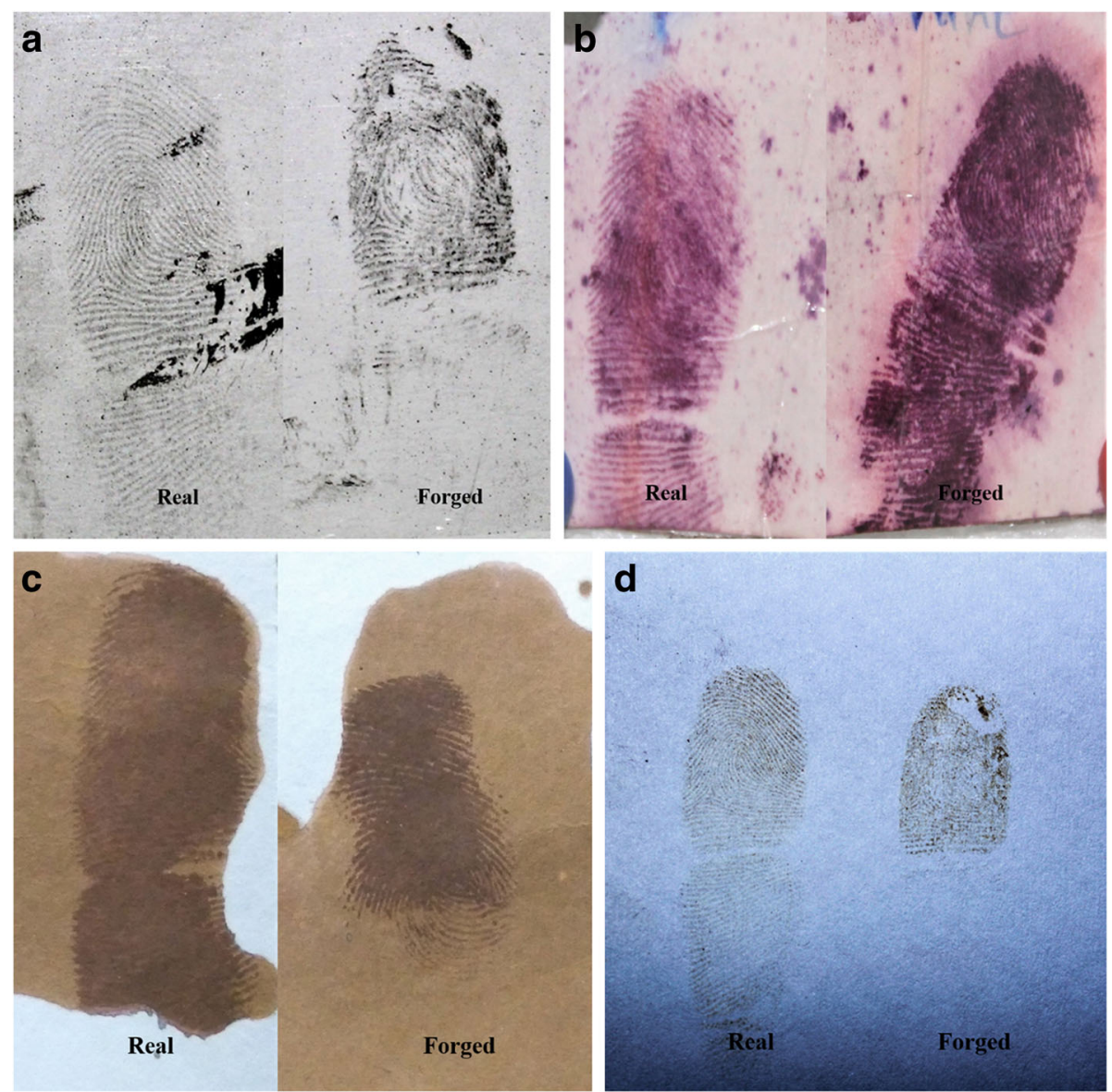

Fig. 1 a Analysis of real and forged fingerprints with black powder (no colour difference observed). b Analysis of real and forged fingerprints with ninhydrin (no colour differentiation). c Analysis of real and forged fingerprints with $\mathrm{AgNO}_{3}$ (no colour differentiation). d Analysis of real and forged fingerprints with iodine fuming (no colour differentiation)

Table 2 The performance of various methods for development of fingerprints, and distinction between real and forged fingerprints

\begin{tabular}{|c|c|c|c|}
\hline Methods & $\begin{array}{l}\text { Real fingerprint } \\
\text { development }\end{array}$ & $\begin{array}{l}\text { Forged fingerprint } \\
\text { development }\end{array}$ & $\begin{array}{l}\text { Colour differentiation between } \\
\text { real and forged fingerprints }\end{array}$ \\
\hline Powder method & $\checkmark$ & $\checkmark$ & $x$ \\
\hline Ninhydrin & $\checkmark$ & $\checkmark$ & $x$ \\
\hline $\mathrm{AgNO}_{3}$ & $\checkmark$ & $\checkmark$ & $x$ \\
\hline lodine fuming & $\checkmark$ & $\checkmark$ & $x$ \\
\hline Powder method followed by $C$ stain & $\checkmark$ & $\checkmark$ & $\checkmark$ \\
\hline Ninhydrin followed by C stain & $\checkmark$ & $\checkmark$ & $\checkmark$ \\
\hline $\mathrm{AgNO}_{3}$ followed by $\mathrm{C}$ stain & $\checkmark$ & $\checkmark$ & $x$ \\
\hline lodine fuming followed by C stain & $\checkmark$ & $\checkmark$ & $\checkmark$ \\
\hline C stain followed by powder method & $\checkmark$ & $\checkmark$ & $x$ \\
\hline C stain followed by ninhydrin & $\checkmark$ & $\checkmark$ & $x$ \\
\hline C stain followed by $\mathrm{AgNO}_{3}$ & $x$ & $x$ & $x$ \\
\hline C stain followed by iodine fuming & $\checkmark$ & $\checkmark$ & $\checkmark$ \\
\hline
\end{tabular}



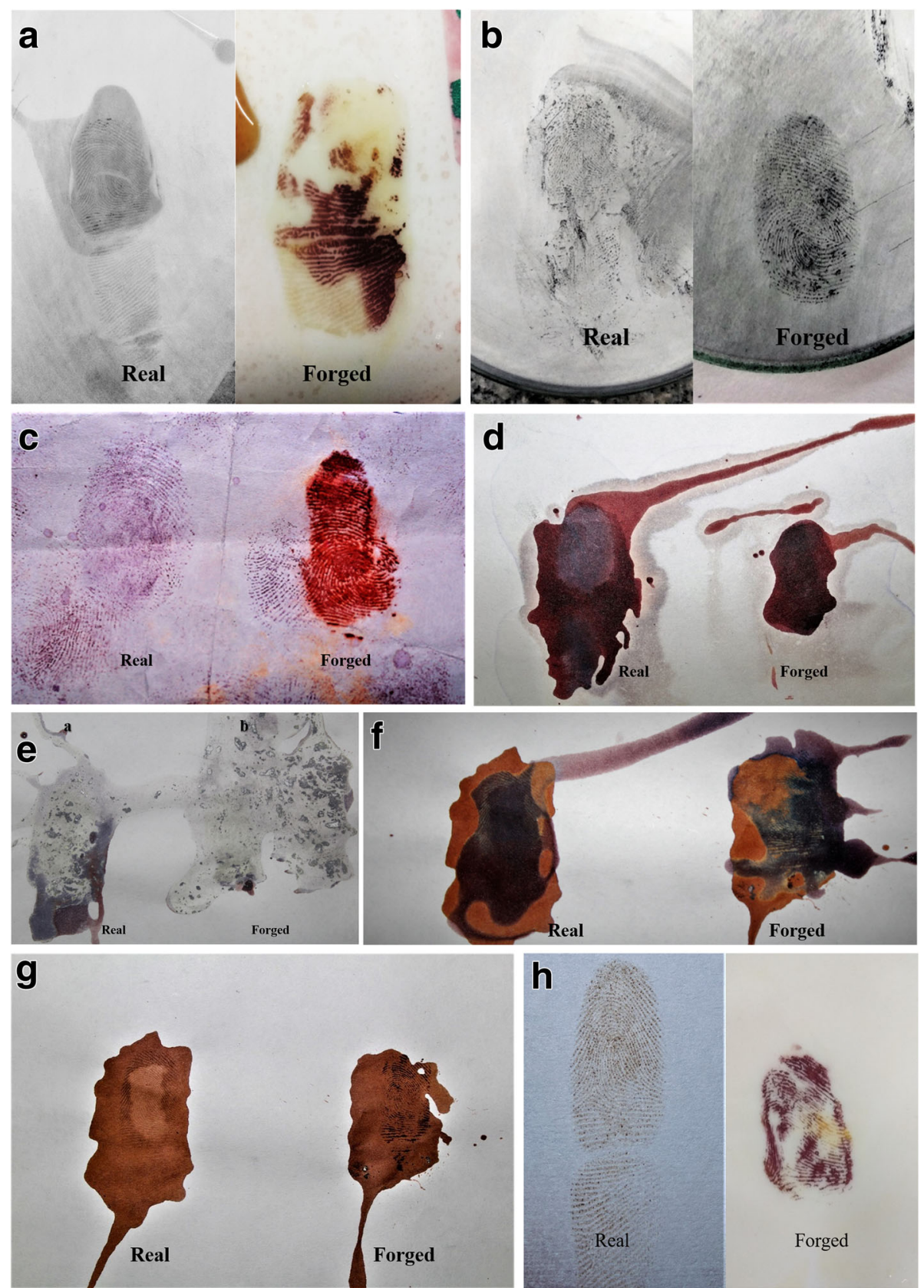

Fig. 2 a Powder method followed by C stain method (colour difference between real and forged fingerprints observed). b C stain followed by powder method (no colour difference between real and forged fingerprints). $\mathbf{c}$ Ninhydrin followed by $\mathrm{C}$ stain (colour difference between real and forged fingerprints observed). $\mathbf{d} \mathrm{C}$ stain followed by ninhydrin (background staining observed). e $\mathrm{AgNO}_{3}$ followed by $\mathrm{C}$ stain (destruction of fingerprints observed). $\mathbf{f} \mathrm{C}$ stain followed by $\mathrm{AgNO}_{3}$ (colour difference between real and forged fingerprints observed). $\mathbf{g}$ lodine fuming followed by $\mathbf{C}$ stain (colour difference between prints and background staining observed). $\mathbf{h} \mathrm{C}$ stain followed by iodine fuming (colour difference between real and forged fingerprints observed)

fingerprints if their development is delayed. The efficacy of $\mathrm{C}$ stain method was observed at different time intervals. A porous paper was used as substrate, and fingerprints were stored at room temperature for different time durations ( 1 day to 4 weeks). The latent fingerprints were then qualitatively analysed with the conventional methods followed by C stain method for development of fingerprints.

\section{Results}

The real and fortified PVA-based fingerprints were developed by conventional methods (powder method, 
ninhydrin, $\mathrm{AgNO}_{3}$, iodine fuming) (Fig. 1a-d), and no difference was observed between real and forged fingerprints (Table 2). On the other hand, $\mathrm{C}$ stain method successfully developed forged fingerprints on both porous and non-porous surfaces, whereas it failed to develop real fingerprints on any surface. The forged fingerprints developed using $\mathrm{C}$ stain appeared in red colour. When $C$ stain was used in combinations with the conventional methods, forged fingerprints appeared in pink colour.

Various combinations of $\mathrm{C}$ stain method with the conventional methods were considered (Table 1) to analyse the impact of these combinations on development and differentiation of fingerprints (Table 2).

- Powder method followed by C stain (Table 1 (1.1)): Powder method developed both fingerprints (real and forged) but failed to differentiate them. After application of $C$ stain, reddish brown colour was observed in forged fingerprints; however, real fingerprints remained unchanged (Fig. 2a).

- C stain followed by powder method (Table 1 (1.2)): When $C$ stain applied on the latent fingerprints, only forged fingerprints were developed. Subsequent application of powder method successfully developed both real and forged fingerprints with black appearance. Thus, prior application of $\mathrm{C}$ stain method did not pose any hindrance in the development of these prints by powder method (Fig. 2b).

- Ninhydrin followed by C Stain (Table 1 (1.3)): Both real and forged fingerprints developed well by ninhydrin, but no differentiation was observed. Subsequent application of $\mathrm{C}$ stain method produced clear differentiation in colour change (purple to red) in forged fingerprint, and no such change was observed in real fingerprints (Fig. 2c).

- C stain followed by ninhydrin (Table 1 (1.4)): When C stain was applied on latent fingerprints, only forged fingerprints were developed. On application of ninhydrin, the $\mathrm{C}$ stain-treated fingerprints got stained with no analyzable impression of fingerprints (Fig. 2d).

- $\mathrm{AgNO}_{3}$ followed by C stain (Table 1 (1.5)): Application of $\mathrm{C}$ stain on $\mathrm{AgNO}_{3}$-treated fingerprints resulted in the complete destruction of both real and forged fingerprints (Fig. 2e).

- C stain followed by $\mathrm{AgNO}_{3}$ (Table 1 (1.6)): After application of $\mathrm{AgNO}_{3}$, the real fingerprints appeared

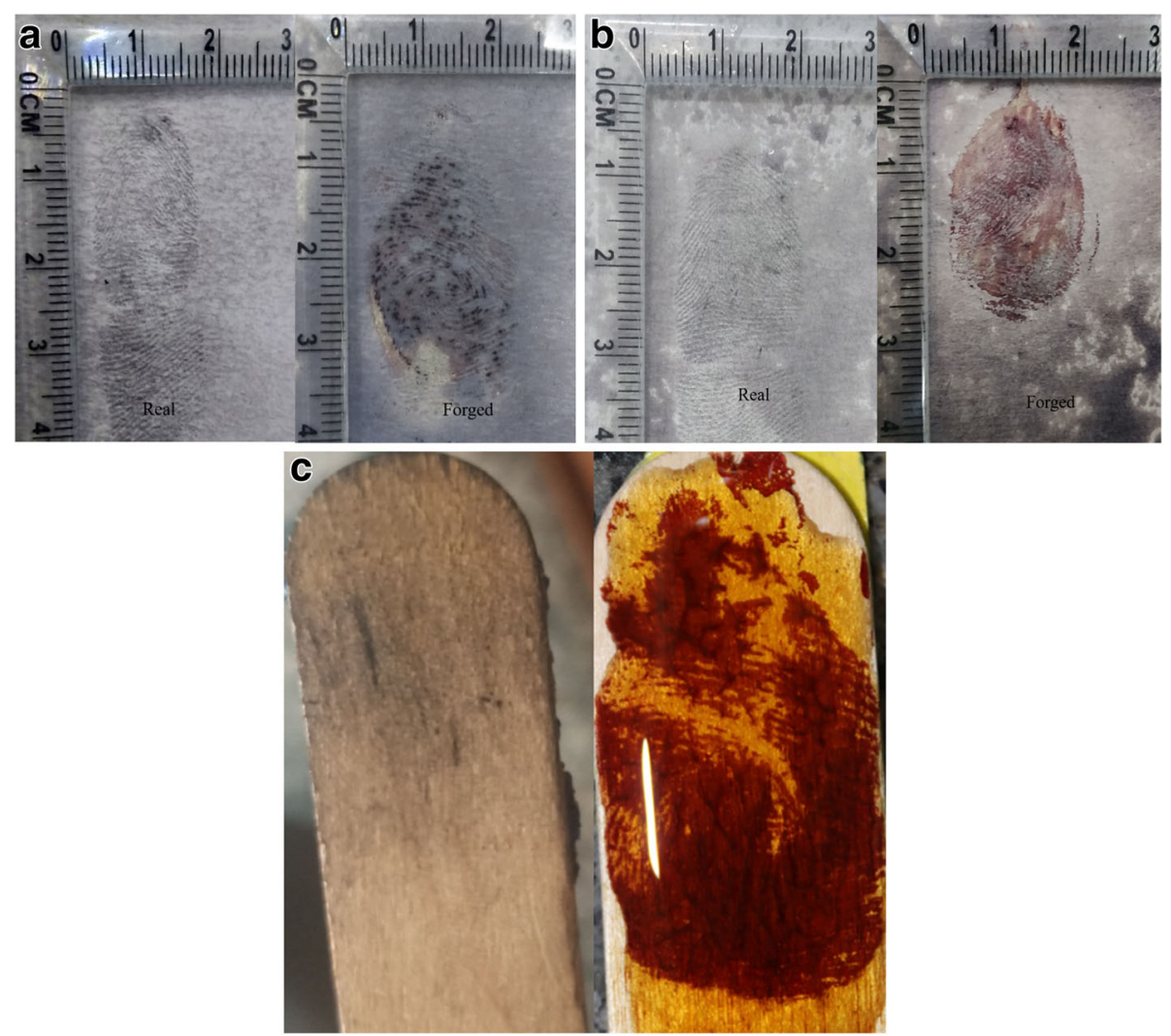

Fig. 3 a Analysis of real and forged fingerprints on porous surface (paper) with black powder followed by $C$ stain method. $\mathbf{b}$ Analysis of real and forged fingerprints on porous surface (cardboard) with black powder followed by $\mathrm{C}$ stain method. c Analysis of real and forged fingerprints on porous surface (wood) with black powder followed by C stain method 
brownish black whereas forged fingerprints appeared to be greenish brown. However, the quality of developed fingerprints was not reasonably good (Fig. 2f).

- Iodine fuming followed by C stain (Table 1 (1.7)): Iodine fumes developed both real and forged fingerprints due to the presence of fatty acids and $\mathrm{NaCl}$. Ridges in forged fingerprints appeared to be darker than real fingerprints when $\mathrm{C}$ stain was applied on these pretreated fingerprints. However, this combination resulted in background staining (Fig. 2g).

- C stain followed by iodine fuming (Table 1 (1.8)): This combination worked well as developed fingerprints were of good quality, and a clear distinction between real and forged fingerprint was observed. Forged fingerprints appeared darker than the real fingerprints (Fig. 2h).

The impact of surface on development of forged fingerprint by $\mathrm{C}$ stain was analysed on porous surfaces (paper, cardboard and wood) and non-porous surfaces (chromogenic paper, plastic bottle, glass). The $\mathrm{C}$ stain method efficiently differentiated forged fingerprints from real fingerprints on paper (Fig. 3a) and cardboard (Fig. 3b), but no development of real fingerprints was observed on wood (Fig. 3c) due to its high porosity. Background staining was observed on all these substrates. The technique worked efficiently on non-porous surfaces (chromogenic paper (Fig. 4a), plastic bottle (Fig. 4b) and glass (Fig. 4c)), and colour differentiation was observed between real and forged fingerprints.

To analyse the efficacy of $\mathrm{C}$ stain method with forged fingerprints prepared with clay and plaster of Paris (PoP), the stain was applied on these latent prints, and as expected, no differentiation between real and forged fingerprints was observed.

The impact of aging of fingerprints on their development and differentiation was analysed on real and forged fingerprints stored at room temperature for different time durations ( 1 day to 4 weeks). No qualitative variation was observed after 1 week of storage, and both real and forged fingerprints were developed and differentiated efficiently by combination of powder method and $\mathrm{C}$ stain method (Fig. 5a). Similar results were observed even after 2 weeks of storage (Fig. 5b). However, real fingerprints were partially developed after 3 weeks, and no development was seen after 4 weeks. Forged fingerprints were developed completely even after 4 weeks (Fig. 5c, d).

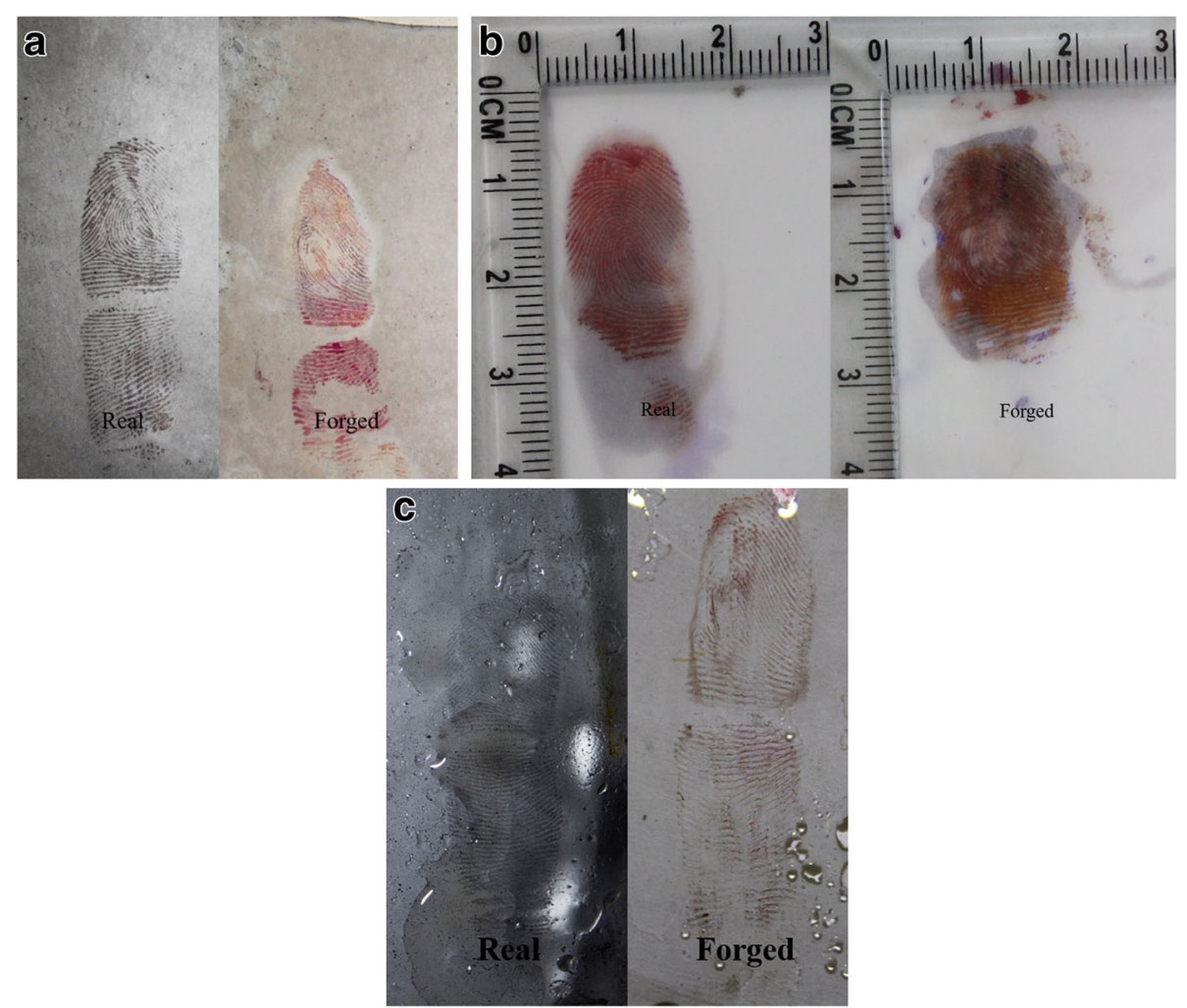

Fig. 4 a Analysis of real and forged fingerprints on non-porous surface (chromogenic paper) with black powder followed by $\mathrm{C}$ stain method. $\mathbf{b}$ Analysis of real and forged fingerprints on non-porous surface (plastic bottle) with black powder followed by $\mathrm{C}$ stain method. $\mathbf{c}$ Analysis of real and forged fingerprints on non-porous surface (glass) with black powder followed by C stain method 


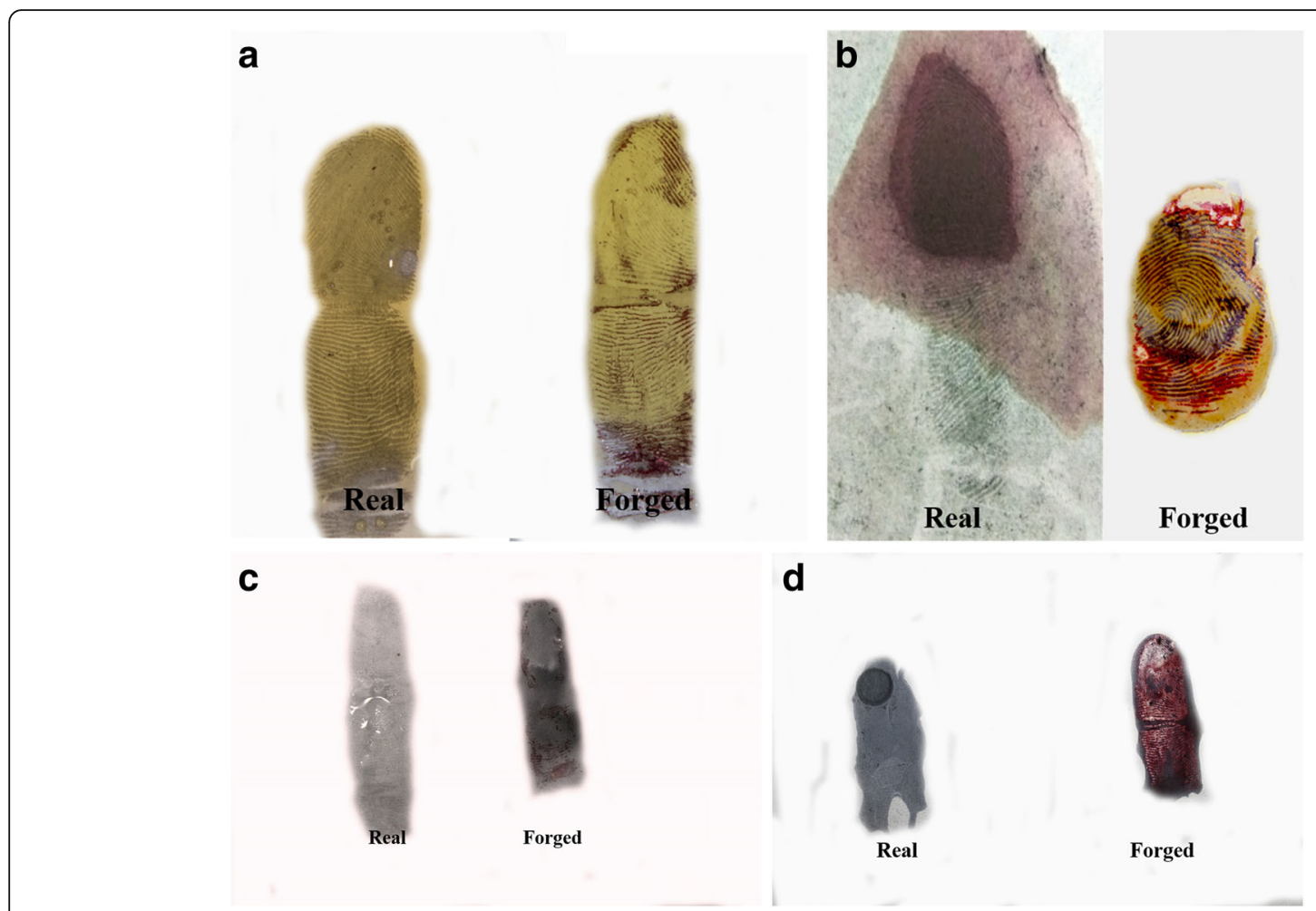

Fig. 5 a Fingerprints development and differentiation after 1 week (black powder followed by C stain method). b Fingerprints development and differentiation after 2 weeks (black powder followed by C stain method). c Fingerprints development and differentiation after 3 weeks (black powder followed by $\mathrm{C}$ stain method). $\mathbf{d}$ Fingerprints development and differentiation after 4 weeks (black powder followed by $\mathrm{C}$ stain method)

\section{Discussion}

Use of forged fingerprints is one of the biggest challenges for the criminal justice system in the absence of their credible detection. Such a situation may lead to false implication of an innocent person. There are a large number of famous and media highlighted cases (David Pearce (1938), Nedelkoff (1940), Harry Oakes (1943), William DePalma (1968), Mickelberg (1980), etc.) where conventional methods failed to differentiate real and forged fingerprints (Champod and Espinoza 2014; Marcel et al. 2014). Another misuse of fraudulent fingerprints is to avoid identification by producing a friction ridge skin having no association with a known fingerprint, either because of it being a mirror image of a genuine fingerprint or a randomly created fingerprint. Carlson (1920) stressed on the risks posed by casting materials which can be used to produce marks with natural secretion and blood. According to the literature, several approaches have been used to ascertain genuineness of fingerprints on the basis of background noise, overall shape of mark, unexpected appearance of the ridges, presence of air bubbles and absence of visible sweat pores. However, their reliability for differentiation has diminished over time (Champod and Espinoza 2014). In case of fortified PVA-based fingerprints, all the known methods fail to differentiate them from the real ones. Thus, the present study is a step towards addressing this problem. In this study, $\mathrm{C}$ stain method is shown to be an effective approach for identification of fortified PVA based forged fingerprints.

This novel protocol differentiated forged fingerprints from the real ones, in terms of colour difference. Its performance was evaluated on porous and non-porous surfaces. While it performed well on non-porous surfaces, $C$ stain reacted with the substrate on porous surfaces and darkened the surface resulting in poor visibility of the developed fingerprint. The $\mathrm{C}$ stain method when applied just after the conventional methods, it produces encouraging results. However, its direct application may lead to alteration of the real fingerprints making it difficult to develop fingerprints by the conventional methods. This method has a limitation in case of ligninbased porous surfaces which get stained with the chemical used and makes fingerprint analysis difficult. If lignin is present in the substrate, $C$ stain method produces a yellow colour on the surface (Adamopoulos and Oliver 2006).

The real and forged fingerprints preserved at room temperature were developed at regular intervals up to 4 weeks to analyse impact of aging of these fingerprints on their development. Partial development of real fingerprints was seen after 3 weeks, and no development was 
observed after 4 weeks, possibly due to degradation of amino acids and salts present in these fingerprints. Forged fingerprints were developed completely even after 4 weeks due to non-degradation of PVA present in these fingerprints.

\section{Conclusion}

On a crime scene, a criminal may leave forged fingerprints to hide his/her identity or to mislead investigators. In such cases, it is difficult to ascertain whether the fingerprints lifted from the crime scene are real or forged prints transplanted by the criminal. In this study, a novel protocol is developed to differentiate forged fingerprints from the real ones, in terms of colour difference. $C$ stain method is an effective technique for distinguishing forged fingerprints from the real ones. It works as a distinction tool even when used in combination with existing development methods. Further, this new technique has been found to be effective irrespective of the time delay in developing fingerprints.

\section{Abbreviations}

PVA: Polyvinyl acetate; $\mathrm{AgNO}_{3}$ : Silver nitrate; PoP: Plaster of Paris; $\mathrm{C}$ stain: Chemical stain

\section{Acknowledgements}

The author(s) would like to acknowledge the support provided under the DST-FIST Grant No.SR/FST/PS-1/2019/68 of Govt. of India. A special thanks to Mr. Kuljeet Yadav, Mr. Shivam Saini, Mr. Naveen Dhull and Miss Taruna Lodhi for laboratory support.

\section{Declaration}

We hereby declare that the matter embodied in this study and entitled "Novel C stain-based chemical method for differentiating real and forged fingerprints" is our original work. Further, we would like to state that the work is not based or reproduced from any existing work already done by any other person for any other purpose.

\section{Authors' contributions}

Mr. Sameer did the experimentation, literature survey and manuscript designing. Prof. A K Yadav advised in manuscript preparation and study design. Dr. Bhuvnesh Yadav did the literature survey and manuscript preparation. The authors read and approved the final manuscript.

\section{Funding}

Not for this particular research.

\section{Availability of data and materials}

With authors.

\section{Ethics approval and consent to participate}

Not applicable

\section{Consent for publication}

Not applicable

\section{Competing interests}

The authors declare that they have no competing interests.

\section{Author details}

${ }^{1}$ Department of Chemistry, Biochemistry and Forensic Science, Amity School of Applied Sciences, Amity University Haryana, Gurugram, Haryana 122413, India. ${ }^{2}$ Amity School of Applied Sciences, Amity University Haryana,

Gurugram, Haryana 122413, India.
Received: 25 October 2019 Accepted: 3 June 2020

Published online: 15 June 2020

\section{References}

Adamopoulos S, Oliver JV (2006) Fiber composition of packaging grade papers as determined by the Graff " $\mathrm{C}$ " staining test. Wood Fiber Sci 38:567-575

Bassam BJ, Caetano-Anolles G, Gresshoff PM (1991) Fast and sensitive silver staining of DNA in polyacrylamide gels. Anal Biochem 196(1):80-83. https:// doi.org/10.1016/0003-2697(91)90120-I

Bonebreak GC (1976) Fabricating fingerprint evidence. Identification News 26(10): $3-13$

Carlson M (1920) Fingerprints can be forged. Va Law Reg 5(10):765-768

Champod C, Espinoza M (2014) Forgeries of fingerprints in forensic science. In: Marcel S, Nixon M, Li S (eds) Handbook of biometric anti-spoofing. Advances in Computer Vision and Pattern Recognition. Springer, London, pp 13-34. https://doi.org/10.1007/978-1-4471-6524-8_2

Choudhari A (2018) Govt to keep check on location of college biometric machines. The Times of India. Available via DIALOG. https://timesofindia. indiatimes.com/city/nagpur/govt-to-keep-check-on-location-of-collegebiometric-machines/articleshow/65554823.cms. Accessed 27 Aug 2019

Geller B, Almog J, Margot P (2001) Fingerprint forgery-a survey. J Forensic Sci 46(3):731-733. https://doi.org/10.1520/jfs15033j

Harvey CJ, LeBouf RF, Stefaniak AB (2010) Formulation and stability of a novel artificial human sweat under conditions of storage and use. Toxicol in Vitro 24(6):1790-1796. https://doi.org/10.1016/j.tiv.2010.06.016

Jasuja OP, Kaur A, Kumar P (2012) Fixing latent fingermarks developed by iodine fuming: a new method. Forensic Sci Int 223(1-3):e47-e52

Jasuja OP, Singh G (2009) Development of latent fingermarks on thermal paper: preliminary investigation into use of iodine fuming. Forensic Sci Int 192(1-3): e11-e16. https://doi.org/10.1016/j.forsciint.2009.08.005

Lee H, Gaensslen RE (2010) Methods of latent fingerprint development. In: Lee HC, Gaensslen RE (eds) Advances in fingerprint technology, 2nd edn. CRC Press, Boca Raton. https://doi.org/10.1201/9781420041347.ch4

LeGrand C (2010) Another look at a Bahamian mystery: the murder of Sir Harry Oakes. Int J Bahamian Stud 16:92-101. https://doi.org/10.15362/ijbs.v16i0.126

Lennard CJ, Margot PA, Stoilovic M, Warrener RN (1986) Synthesis of ninhydrin analogues and their application to fingerprint development: preliminary results. J Forensic Sci Soc 26(5):323-328. https://doi.org/10.1016/S0015$7368(86) 72510-3$

Marcel S, Nixon MS, Li SZ (2014) Handbook of biometric anti-spoofing-trusted biometrics under spoofing attacks. Springer-Verlag, New Yark. https://doi.org/ 10.1007/978-1-4471-6524-8

Oden S, Von Hofsten B (1954) Detection of fingerprints by the ninhydrin reaction. Nature 173:449-450. https://doi.org/10.1038/173449a0

Qazi M (2017). You will be glued to this: Mumbai college's students trick biometric system. Hindustan Times. Available via DIALOG. https://www. hindustantimes.com/mumbai-news/you-will-be-glued-to-this-mumbaicollege-s-students-trick-biometric-system/story-W64f1jdMtecxKDml2Dakel. html. Accessed 15 May 2019.

Saldanha A (2018) From cheating banks to faking identity, Aadhaar frauds peak in 2018 : report. Business Standard. Available via DIALOG. https://www. business-standard.com/article/economy-policy/from-cheating-banks-tofaking-identity-aadhaar-frauds-peak-in-2018-report-118052300151_1.html. Accessed 26 Sept 2019

Sodhi GS, Kaur J (2001) Powder method for detecting latent fingerprints: a review. Forensic Sci Int 120(3):172-176. https://doi.org/10.1016/S03790738(00)00465-5

Somanchi A (2018) Aadhaar fraud is not only real, but is worth more closely examining. The Wire. Available via DIALOG. https:/thewire.in/economy/ aadhaar-fraud-uidai. Accessed 26 September 2019.

Sousedik C, Busch C (2014) Presentation attack detection methods for fingerprint recognition systems: a survey. IET Biometrics 3(4):219-233. https://doi.org/10. 1049/iet-bmt.2013.0020

\section{Publisher's Note}

Springer Nature remains neutral with regard to jurisdictional claims in published maps and institutional affiliations. 